\title{
Evaluación in vitro de tres solventes de gutapercha
}

\author{
ARTículo ORIGINAL
}

In vitro evaluation of three gutta-percha solvents

\section{Resumen}

El objetivo de ésta investigación fue evaluar la solubilidad de la gutapercha en tres solventes usados en endodoncia: xylol de uso tradicional y los aceites esenciales: eucaliptol y aceite de naranja, de uso reciente, dada la frecuente necesidad de remover las obturaciones del conducto en los procedimientos de retratamiento radicular. Ciento veinte muestras de gutapercha fueron preparadas en forma estandarizada en pequeños cilindros, siendo divididos equitativamente en cuatro grupos para su inmersión en los tres solventes de experimentacion y en agua destilada (grupo control) durante 2,5 y 10 minutos, a temperatura ambiente. La efectividad solvente fue registrada en gramos de pérdida de peso, calculando la diferencia entre el peso pre inmersión y post inmersión de las muestras, para cada tiempo experimental. Los resultados fueron analizados y comparados mediante la prueba de Tukey $(\mathrm{p}<0.05)$. Bajo las condiciones del presente estudio se concluye, que todos los solventes experimentales fueron efectivos para disolver la gutapercha. El xylol presentó efectos solventes superiores en todos los tiempos de experimentación. El aceite de naranja y eucaliptol presentaron efectos solventes similares.

Palabras clave: Endodoncia, Gutapercha, Solventes orgánicos, Aceite de naranja

\section{Abstract}

The purpose of this study was to evaluate the solubility of gutta-percha in three solvents used in endodontics: xylol of traditional use, and the essential oils: eucalyptol and orange oil, of recent use, given the frequent need to remove the obturation material in radicular retreatment procedures. One hundred twenty samples of gutta-percha were prepared in a standardized way in small cylinders, being divided equally into four groups for immersion in three experimental solvents and distilled water (control group) during 2.5 and 10 minutes at room temperature. The solvent effect was recorded in grams of weight loss, calculating the difference between pre and post-immersion samples weight for each experimental time. The results were analyzed and compared by Tukey test $(\mathrm{p}<0.05)$. Under the conditions of this study it is concluded that, all experimental solvents were effective to dissolve gutta-percha. Xylol showed superior solvent effects in all experimental times. Orange oil and eucalyptol presented similar solvent effects. Key words: endodontics. Gutta-percha. Organic solvents. Orange oil

\section{Martha Elena Pineda Mejía ${ }^{1}$, Elmo Sigifredo Palacios Alva ${ }^{2}$, Liliana Ángela Terán Casafranca ${ }^{1}$, María Elena Núñez Lizárraga ${ }^{3}$, Waldo Ernesto Gloria Zevallos ${ }^{1}$, Tulio Abuhadba Hoyos ${ }^{1}$}

\footnotetext{
1 Dpto. Académico: Estomatología Rehabilitadora. Fac. Odontología - UNMSM.

2 Dpto. Académico: Estomatología Médico Quirúrgico. Fac. Odontología - UNMSM.

3 Dpto. Académico: Estomatología Pediátrica. Fac. Odontología - UNMSM.
}

Correspondencia:

Mg. Martha Elena Pineda Mejía,

Av. Venezuela cdra. 34. Ciudad Universitaria, Lima 01.

E-mail: marthapime@hotmail.com

Fecha de recepción: 18-04-11

Fecha de aprobación: 9-08-11

\section{Introducción}

La gutapercha es el material de más frecuente uso en el paso de obturación del conducto radicular. Este material termoplástico cumple con los principales requisitos de un material de relleno del conducto radicular, uno de los cuales está referido a ser un material de fácil remoción en casos de retratamiento endodontico. Los métodos más usados para llevar a cabo la desobturación radicular son: mecánico, térmico, químico, o también una asociación de ellos, aunque también puede ser usado el método a base de instrumentos especiales como son los ultrasónicos. ${ }^{1,2,7,9}$

Los conos de gutapercha endodónticos están compuestos de una resina vegetal, de la cual precisamente proviene su nombre, estos conos pueden ser ablandados por solventes químicos. ${ }^{11}$

Entre los solventes orgánicos que son usados con mayor frecuencia en endodoncia tenemos: cloroformo, xylol, ha- lotano, eucalyptol, turpentine, y aceite de naranja. $1,4,5,6,11,15,17$

La gutapercha puede ser fácilmente removida con el uso de un solvente orgánico o con un instrumental calentado. Sin embargo, estas sustancias parecen mostrar diferentes grados de éxito en la disolución y remoción de la obturación de gutapercha presente en el conducto radicular.

Los solventes orgánicos han sido usados desde hace mucho tiempo atrás como método auxiliar o principal para la remoción de la gutapercha, considerándoseles como las sustancias químicas más efectivas para disolver el relleno de material endodóntico.

El cloroformo y el xileno son dos solventes comúnmente usados, pero la US FOOD ADMINISTRATION prohíbe el uso de cloroformo debido a su potencial carcinogénico. Por el contrario, el xileno está disponible actualmente para uso clínico, y no es considerado carcinogénico, aunque resulta ser muy tóxico para los tejidos. ${ }^{3,13,18}$

La gutapercha también es soluble en aceites esenciales. Algunos de ellos han sido reportados como seguros y útiles para este propósito, por ejemplo, los aceites esenciales: eucalytus (eucalytol) y árbol de pino (turpentine $)^{14}$. De acuerdo con Pécora y col. ${ }^{10}$ 1992, el aceite de naranja actúa sobre la gutapercha en la misma manera que lo hace el xylol, sin la presentación de ningún efecto deletéreo.

En estos días, los procedimientos de retratamiento han cobrado mucha importancia en endodoncia, reemplazando métodos quirúrgicos tradicionales. Los diferentes solventes han sido por mucho tiempo usados para desobturar el conducto radicular. Sus propiedades deberían ser tomadas en consideración con relación a su efectividad en la disolución del material de obturación radicular. 
El propósito de este estudio es evaluar comparativamente la eficiencia de disolución de tres diferentes solventes orgánicos sobre la gutapercha en tres intervalos de tiempo. Los objetivos esla pérdida de peso de la gutapercha después de su inmersión en tres diferentes solventes orgánicos: xylol, eucalyptol y aceite de naranja, en los periodos de tiempo: $2 \mathrm{~min}, 5 \mathrm{~min}$ y $10 \mathrm{~min}$; y b) Realizar un análisis comparativo de la eficiencia solvente de las tres sustancias en experimentación.

\section{Material y método}

En esta investigación se usaron los coEndomedic (SURE DENT CORPORATION COREA, R.S.E-8518-IMN. Corea), y los solventes eucalyptol (EGEO S.R.L. Argentina), xylol (PRObia), y el aceite de naranja (MAQUIRA INDUSTRY ODONTOLOGICAL PRODUCTS LTDA, Maringa, Brazil). El agua destilada químicamente pura (DIAMEDSA, Distribuidores y Abastecederoes Médicos. Lima, Perú), fue usada como grupo control no solvente.

La muestra quedó constituida por 120 pequeños cilindros de $8 \mathrm{~mm}$ de diámetro y $2 \mathrm{~mm}$ de alto, los conos de gutapercha fueron calentados y condensados dentro de ellos, luego dejados a temperatura ambiente por 48 horas. El exceso de material de la superficie fue retirado con una hoja de bisturí. Luego los discos fueron divididos en 4 grupos de 30 cada uno, distribuidos en relación al solvente y al tiempo de la siguiente manera:

\begin{tabular}{lccc}
\hline & $\begin{array}{c}2 \\
\text { min }\end{array}$ & $\begin{array}{c}5 \\
\min \end{array}$ & $\begin{array}{c}10 \\
\text { min }\end{array}$ \\
\hline Xylol & 10 & 10 & 10 \\
\hline Eucaliptol & 10 & 10 & 10 \\
\hline $\begin{array}{l}\text { Aceite de } \\
\text { naranja }\end{array}$ & 10 & 10 & 10 \\
\hline Agua destilada & 10 & 10 & 10 \\
\hline
\end{tabular}

Las muestras fueron pesadas en gramos en una balanza digital (OHAUS modelo precision estándar) considerando los registros hasta tres decimales. Este pesaje se realizó en los siguientes momentos: antes de la inmersión en el solvente, a los $2 \mathrm{~min}, 5 \mathrm{~min}$, y $10 \mathrm{~min}$ después de la inmersión en el solvente.

Para lo cual los discos fueron colocados, en un recipiente de vidrio, de modo pecíficos fueron: a) Determinar in vitro nos de gutapercha de la marca comercial QUIDENT S.A. Antioquia. Colom-

que ambas superficies del disco quedaron expuestas a la sustancia.

Después del periodo de inmersión las muestras fueron lavadas con agua destilada y dejadas a secar a una temperatura de $37^{\circ} \mathrm{C}$ en una estufa por 24 horas.

La eficiencia de disolución de los solventes estuvo dada por la diferencia de peso entre el peso original y el peso registrado post inmersión en el solvente respectivo.

Se hallaron valores promedios, desviación estándar de la pérdida de peso en cada intervalo de tiempo, para cada grupo experimental. Las diferencias de disolución entre los diferentes valores hallados se hicieron mediante la prueba de Tukey, para muestras independientes, en los diferentes tiempos experimentales a una significancia estadística del $95 \%$.

Tabla 1. Promedio y desviación estándar de la solubilidad de la gutapercha en tres solventes orgánicos expresada en gramos de pérdida de peso

\begin{tabular}{llccc}
\hline \multicolumn{1}{c}{ Tiempo } & Aceite de naranja & Eucaliptol & Xylol & Agua destilada \\
\hline 2 minutos & $0.005 \pm 0.0035$ & $0.007 \pm 0.0025$ & $0.031 \pm 0.0374$ & $0.001 \pm 0.0013$ \\
5 minutos & $0.011 \pm 0.0059$ & $0.011 \pm 0.0067$ & $0.032 \pm 0.1017$ & $0.001 \pm 0.0009$ \\
10 minutos & $0.020 \pm 0.0110$ & $0.024 \pm 0.0123$ & $0.066 \pm 0.0134$ & $0.001 \pm 0.0007$ \\
\hline
\end{tabular}

$\mathrm{F}=42.11(\mathrm{p}=0.000)$

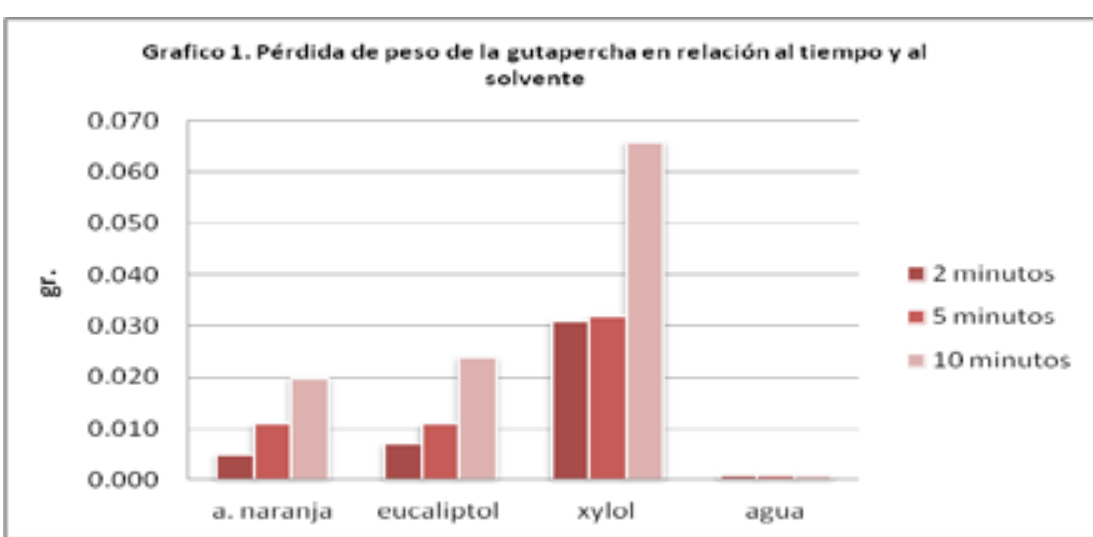

Tabla 2. Comparación múltiple: Prueba de Tukey (95\% Simultaneous Confidence Intervals)

\begin{tabular}{|l|l|l|l|}
\hline Compared groups & & lower & upper \\
\hline Xylol 2 min vs & Eucaliptol $2 \mathrm{~min}$ & $(0.020690$, & $0.049710)^{*}$ \\
\hline Xylol 5 min vs & Eucaliptol $5 \mathrm{~min}$ & $(0.006290$, & $0.035310)^{*}$ \\
\hline Xylol 10 min vs & Eucaliptol $10 \mathrm{~min}$ & $(0.027290$, & $0.056310)^{*}$ \\
\hline & & & \\
\hline Xylol 2 min vs & Naranja $2 \mathrm{~min}$ & $(0.022590$, & $0.051610)^{*}$ \\
\hline Xylol 5 min vs & Naranja $5 \mathrm{~min}$ & $(0.006590$, & $0.035610)^{*}$ \\
\hline Xylol 10 min vs & Naranja $10 \mathrm{~min}$ & $(0.031990$, & $0.061010)^{*}$ \\
\hline & & & \\
\hline Naranja 2 min vs & Eucaliptol 2 min & $(-0.012610$, & $0.016410)$ \\
\hline Naranja 5 min vs & Eucaliptol 5 min & $(-0.014210$, & $0.014810)$ \\
\hline Naranja 10 min vs & Eucaliptol $10 \mathrm{~min}$ & $(-0.009810$, & $0.019210)$ \\
\hline
\end{tabular}


te mayor que el aceite de naranja y eucaliptol en todos los tiempos de inmersión considerados, mientras que entre el aceite de naranja y eucaliptol no hubo diferencias significantes $(p>0.05)$, el agua destilada no promovió alteraciones en la gutapercha.

El análisis de varianza, mediante la prueba $\mathrm{F}$ demostró que existe diferencia significante entre todos los grupos $(\mathrm{p}=0.000)$.

Se hizo el análisis de comparaciones múltiples aplicando la prueba de Tukey (ver Tabla 2), la cual mostró que todos los grupos solventes fueron significativamente mayores que el grupo control con agua. El xylol fue significativamente mayor que el aceite de naranja y eucaliptol en todos los tiempos de inmersión considerados, mientras que entre el aceite de naranja y eucaliptol no hubo diferencias significantes ( $p>0.05)$, el agua destilada no promovió alteraciones en la gutapercha.

\section{Discusión}

La estandarización de los ensayos clínicos concernientes a la solubilidad en general sigue la normativa ISO. Sin embargo, no existe mucha normativa para evaluar los efectos de solventes endodónticos sobre materiales de gutapercha.

Considerando la gran perspectiva de éxito en reintervenciones de gutapercha, el retratamiento llega a convertirse en una conducta clínica conservativa en comparación con algunos procedimientos más radicales tal como la cirugía periapical. La remoción del material de obturación endodóntico del conducto radicular es un requerimiento para el retratamiento.

La gutapercha en conjunto con una variedad de selladores radiculares, es el método de obturación radicular más comúnmente usado. Muchos métodos para remover el material de obturación están disponibles, incluyendo el uso de solventes, el método térmico e instrumentación mecánica, o una combinación de los tres. ${ }^{1,2,7,9}$

La gutapercha puede ser removida sin mayor dificultad con el uso de solventes orgánicos o instrumentos calientes. Nuestros resultados mostraron que el xylol es el material más efectivo para la disolución de la gutapercha comparado con los otros solventes testeados. Eucaliptol, y aceite de naranja mostraron efectos disolventes similares, mientras que el agua no mostró ningún efecto.
Los métodos químicos para la remoción de gutapercha o la descontaminación ${ }^{12}$ han sido usados por mucho tiempo. Sin embargo, las sustancias que son los solventes más efectivos, tiene el mayor potencial de toxicidad. Muchos estudios reportan que incluso sustancias que están lejos del periapice, como por ejemplo la cámara pulpar, pueden alcanzar los tejidos periapicales. ${ }^{16}$

El Xylol y el cloroformo están clasificados como los solventes de mayor capacidad disolutoria de la gutapercha, pero también presentan efectos muy indeseables en los tejidos periapicales, considerados potencialmente carcinogénicos (cloroformo) o neurotóxicos (xileno). ${ }^{18}$

En el presente estudio, el aceite de naranja y el eucaliptol mostraron estadísticamente menor capacidad disolutoria que el xylol $(p>0.05)$, en contraposición a los resultados de Hunter et al. ${ }^{4}$ 1991 y Silva M et al. (2007), pero en concordancia con muchos otros investigadores. ${ }^{3,15,17}$

El uso de aceites esenciales en endodoncia está creciendo, debido a su probada seguridad, biocompatibilidad y no carcinogenicidad. ${ }^{5,6}$ Los resultados de este estudio indican que ambos, el aceite de naranja y el eucaliptol, son apropiados como solventes, para disolver o ablandar la gutapercha, si bien no lo hicieron con la capacidad del xylol, sin embargo, tienen a su favor la biocompatibilidad y no toxicidad.

Los aceites esenciales extraídos de la piel de la naranja, Citrus aurantium, son fáciles de obtener y además su uso es apropiado para abrir rápidamente el conducto radicular, principalmente en obturaciones con cemento de óxido de zinc eugenol, asociado o no con conos de gutapercha. El aceite de naranja es un solvente alternativo excelente comparado con los solventes potencialmente tóxicos, siendo usados ya sea sobre cemento óxido de zinc eugenol o para ablandar o disolver la gutapercha. ${ }^{8,9,10,14}$

El eucaliptol (principal constituyente del aceite de eucalipto) exhibe efectos antibacterianos y propiedades antiinflamatorias, y su potencial de disolución de la gutapercha incrementa significativamente cuando es calentado, ${ }^{9,11}$ si no es calentado, disuelve el material más lentamente.

Más aún, después de vaciar los conductos, este solvente es fácilmente removido por irrigación con soluciones tenso activas tales como el lauril sodio sulfato o similares. ${ }^{10}$
Considerando la existencia de similitudes en la capacidad solvente entre aceites esenciales y de otros solventes orgánicos investigados, nosotros podríamos usar clínicamente eucaliptol y aceite de naranja por espacios de tiempo más prolongados. La miscibilidad y la profundidad de penetración incontrolables de soluciones como el xylol y otros de su tipo, llevan muchas veces a un campo que no es controlable por nosotros, al alcanzar la región apical y causando pericementitis química.

La elección de un solvente ideal para retratamientos endodónticos requiere el establecimiento de un balance entre el nivel de seguridad clínica, el nivel de agresión a los tejidos, y la capacidad química de disolución.

\section{Conclusiones}

Dentro de los alcances de este estudio in vitro, llegamos a las siguientes conclusiones:

1. Todos los solventes orgánicos, objetivo del estudio, fueron efectivos para disolver la gutapercha.

2. El xylol presentó efectos solventes superiores a todos.

3. El aceite de naranja y eucaliptol presentaron efectos solventes similares.

4. Limitar el uso clínico de solventes que presentan efectos tóxicos y carcinogénicos.

\section{Referencias bibliográficas}

1. Bueno CES, Valdrighi L. Efetividade de solventes e de técnicas na desobturação dos canais radiculares: estudo in vitro. Rev Bras Odontol. 1998;55(4):251-5.

2. Friedman S, Stabholz A, Tamse A. Endodontic retreatment - case selection and technique. 3. Retreatment techniques. $J$ Endod. 1990; 16(11):543-9.

3. Görduysus MÖ, Tasman F, Tuncer S, Etikan I. Solubilizing efficiency of different gutta-percha solvents: a comparative study. J Nihon Univ Sch Dent. 1997; 39(3):133-5.

4. Hunter RK, Doblecki W, Pelleu GB. Halothane and eucalyptol as alternatives to chloroform for softening gutta-percha. J Endod. 1991; 17(7):310-2.

5. Kaplowitz GJ. Evaluation of gutta-percha solvents. $J$ Endod. 1990;16(11):539-40. 
6. Kaplowitz GJ. Evaluation of the ability of essential oils to dissolve gutta-percha. J Endod. 1991; 17(9):448-9.

7. Ladley RW, Campbell AD, Hicks ML, Li SH. Effectiveness of halothane used with ultrasonic or hand instrumentation to remove gutta-percha from the root canal. $J$ Endod. 1991; 17(5):221-4.

8. Martos J, Gastal MT, Sommer L, Lund RGL, Del Pino FAB, Osinaga PWR. Dissolving efficacy of organic solvents on root canal sealers. Clin Oral Invest. 2006; 10(1):50-4.

9. Oyama KON, Siqueira EL, Santos $M$. In vitro study of effect of solvent on root canal retreatment. Braz Dent J. 2002; 13(3):208-11.

10. Pécora JD, Costa WF, Filho DS, Sarti SJ. Apresentação de um óleo essencial, obtido de Citrus aurantium, eficaz na desintegração do cimento de óxido de zinco-eugenol do interior do canal radicular. Odonto. 1992; 1(5):130-2.

11. Pécora JD, Spanó JCE, Barbin EL. In vitro study on the softening of gutta-percha cones in endodontic retreatment. Braz Dent J. 1993; 4(1):43-7.

12. Souza RE, Souza EA, Sousa-Neto $\mathrm{MD}$, Pietro RC. In vitro evaluation of different chemical agents for the decontamination of gutta-percha cones. Pesqui Odontol Bras. 2003; 17(1):75-7.

13. Spangberg L, Langeland K. Biologic effects of dental materials. 1 . Toxicity of root canal filling materials on HeLa cells in vitro. Oral Surg Oral Med Oral Pathol. 1973; 35(3):402-14.
14. Spanó JCE, Barbin EL, Bonini A, Pécora JD. Eficácia dos óleos essenciais na desobturação dos canais radiculares. ROBRAC. 1995; 5(14):25-8.

15. Tamse A, Unger U, Metzger Z, Rosenberg M. Gutta-percha solvents a comparative study. J Endod. 1986; 12(8):337-9.

16. Walton RE. Intracanal medications. Dent Clin North Am. 1984; 28(4):783-96.

17. Wennberg A, Orstavik D. Evaluation of alternatives to chloroform in endodontic practice. Endod Dent Traumatol. 1989; 5(5):234-7.

18. Wourms DJ, Campbell AD, Hicks ML, Pelleu GB Jr. Alternative solvents to chloroform for guttapercha removal. J Endod. 1990; 16(5):224-6. 